ÉGYPTE monde arabe

\section{Égypte/Monde arabe}

30-31 | 1997

Les visions de l'Occident dans le monde arabe

\title{
Marxisme égyptien et marxisme occidental : traduction et idéologie
}

\section{Anwar Moghith}

\section{Q OpenEdition}

\section{Journals}

Édition électronique

URL : https://journals.openedition.org/ema/1600

DOI : 10.4000/ema. 1600

ISSN : 2090-7273

\section{Éditeur}

CEDEJ - Centre d'études et de documentation économiques juridiques et sociales

\section{Édition imprimée}

Date de publication : 30 septembre 1997

Pagination : 71-91

ISSN : 1110-5097

\section{Référence électronique}

Anwar Moghith, « Marxisme égyptien et marxisme occidental : traduction et idéologie », Égypte/Monde arabe [En ligne], 30-31 | 1997, mis en ligne le 08 juillet 2008, consulté le 07 juillet 2022. URL : http:// journals.openedition.org/ema/1600; DOI : https://doi.org/10.4000/ema.1600

Ce document a été généré automatiquement le 7 juillet 2022.

Tous droits réservés 


\title{
Marxisme égyptien et marxisme occidental : traduction et idéologie
}

\author{
Anwar Moghith
}

1 La rencontre entre les Égyptiens et la pensée marxiste coïncide avec le développement de la presse libre pendant la deuxième moitié du XIX ${ }^{\mathrm{e}}$ siècle. Cette presse devait rendre compte des divers événements qui se déroulaient alors en Occident : guerres, alliances, inventions, etc. Certains événements spectaculaires, comme la Commune de Paris, l'assassinat du Tsar, l'entrée des députés socialistes au Reichstag (parlement allemand) et l'assassinat du président français Sadi Carnot, ainsi que l'émergence de nouveaux phénomènes comme les syndicats ouvriers et les grèves, ont incité la presse égyptienne à s'intéresser aux mouvements sociaux. Ceux-ci ont été désignés au départ par leurs noms européens translitérés en arabe: al-sûsyâlist, al-kumyunist et al-nihîlist. Ces courants sont toujours mentionnés ensemble, sans distinction entre eux, et en général, ils sont tous réfutés par un seul argument qui souligne leur opposition à la nature humaine et le retour à l'état animal qu'ils entraînent (Al-Afghânî, 1942 ; Ishâq, 1978 ; alMuqtataf, 1879).

2 Ce recours à la translitération reflète une certaine conviction, chez les écrivains et les rédacteurs de l'époque, selon laquelle les mouvements sociaux seraient des phénomènes proprement occidentaux. Toutefois, cette conviction a été ébranlée par l'impact de plus en plus sensible de l'Occident sur la vie des Égyptiens et par le fait que les penseurs de la Nahda (Renaissance arabe) ont choisi l'Occident comme modèle et comme fin de leur démarche progressiste. Dans ce contexte, le mot du Manifeste communiste selon lequel la bourgeoisie occidentale "crée un monde à son image " (Marx, 1965b, p. 165), décrit parfaitement le fond de la pensée des intellectuels égyptiens de la Nahda qui voit dans la civilisation occidentale l'image de leur propre avenir. La presse ne pouvait donc rester indifférente aux mouvements sociaux car ceux-ci allaient nécessairement concerner l'Égypte. En effet, ces derniers lui apparaissent, tout comme les inventions qui remplissent les pages de la même presse, transculturels et transnationaux. On abandonne alors la translittération pour la recherche de mots arabes pouvant exprimer le sens de ces mouvements. Adîb Ishâq 
choisit provisoirement dans sa revue al-Mahrûsa le terme al-khawârij pour désigner les trois courants d'une manière générale. À l'origine, ce mot désigne un mouvement contestataire du début de l'islam, qui, face aux groupes d'intérêts composés des compagnons du Prophète, revendiquait l'application d'un islam authentique. Ce mot, outre qu'il donne aux mouvements sociaux un aspect religieux qu'ils n'ont pas, ne peut remplacer une traduction. C'est Ahmad Fâris al-Shidyâq qui sera le premier, en 1879, à traduire le mot socialisme par ishtirâkiyya ; un mot qui signifie en arabe "partage ». Ishâq traduit communisme par ibâhiyya qui signifie «licence » ou «libertinage ». La revue al-Muqtataf traduit le mot grève par i'tisâb qui signifie " regroupement agressif $»^{1}$. Le choix de ces mots arabes reflète le désir des traducteurs de créer une certaine répugnance à l'égard des mouvements sociaux. Cette nouvelle approche, qui consiste à abandonner la translittération pour l'usage de mots vernaculaires coïncide avec la volonté de découvrir les traits distinctifs de chaque courant. Une distinction qui fait apparaître le socialisme comme le plus modéré de ces courants. Au cas où il faudrait choisir, il représenterait le moindre mal.

3 La conviction que l'Occident représente le modèle de la modernisation en Égypte a, d'un côté, incité les intellectuels à développer leur critique à l'égard des mouvements sociaux. Un nouvel argument adopté contre ces mouvements ne s'appuie plus sur l'opposition à la nature humaine ou l'oubli de la loi divine ; il s'inspire de la théorie de l'évolution: ces mouvements violent la loi de l'évolution en provoquant un changement brutal. Cet argument a l'avantage de réfuter les mouvements sociaux tout en restant moderniste. D'un autre côté, et du fait même de ce double paradigme de la modernisation (l'Occident comme modèle et l'évolutionnisme appliqué à la société), les courants de pensée véhiculés par les mouvements sociaux en Occident, tels que le socialisme et le communisme, ont commencé à gagner des partisans parmi les intellectuels égyptiens. Ce qui donne lieu à une approche beaucoup plus intéressante et familière, et pousse les intellectuels à traduire des textes appartenant à ces courants.

\section{La traduction des textes marxistes}

Durant les deux premières décennies du $\mathrm{XX}^{\mathrm{e}}$ siècle, le socialisme est dans la presse égyptienne un objet de polémique, abordé en termes vifs et passionnés par les intellectuels. Le socialisme est présenté alors comme une nouvelle et curieuse doctrine. Il est la cause d'événements violents troublant la vie politique internationale, il est un objet sur lequel des écrivains célèbres et des politiciens exercent leur rhétorique pour le réfuter ou en faire l'éloge. Une telle présentation du socialisme, bien que non fondée sur une connaissance réelle de la doctrine, entraîne des réactions d'enthousiasme ou de rejet.

En effet, aucune œuvre n'est, en effet, encore traduite et les divergences au sein du mouvement socialiste restent inconnues. Le marxisme est encore plus méconnu ; Karl Marx est célébré comme fondateur du socialisme scientifique, théoricien socialiste et comme grand philosophe allemand. Pour autant, les intellectuels égyptiens optent pour un socialisme graduel et modéré et rejettent le communisme jugé, trop dangereux : la réforme est mieux admise que la révolution. Malgré l'adhésion du Parti communiste égyptien (PCE) à la III Internationale en 1922, le marxisme n'y est pas présent en tant que théorie, car le parti, trop préoccupé par la lutte syndicale, n'offre ni textes accessibles, ni recherches, ni réflexions à propos des thèses marxistes. 
6 Dans le débat journalistique et littéraire de l'époque, le mot marxisme (al-madhhab almârksî) est peu utilisé. Les mots communisme (al-shuyâ'iyya) et plus tard bolchevisme (al-bulshufiyya) sont courants, non pas pour signifier une théorie, une idéologie ou une vision du monde, mais plutôt comme concepts d'une nouvelle organisation sociale. On parle du communisme comme d'une bonne nouvelle ou d'une menace, on parle de Marx comme d'un messie ou d'un faux messie. Cet aspect mystérieux provient de l'ignorance des idées et des textes marxistes, de l'ignorance de cette infrastructure théorique nécessaire à toute adoption ou réfutation utile et bien construite d'une idée nouvelle. Il est donc important d'accorder à la parution des textes marxistes une grande considération et une attention toute particulière, car elle assure enfin la présence réelle du marxisme en langue arabe.

7 Ces textes sont particulièrement significatifs en ce qui concerne la présence de la culture occidentale au sein de la culture arabo-musulmane. Car le marxisme, avec sa prétention à la validité universelle, invite le traducteur à trouver des contenus à ses concepts à l'intérieur de sa propre culture. Autour de la traduction s'étend un large champ de connaissances où se situent : le traducteur, en tant que personne réelle qui a une certaine situation sociale et qui entre en interaction avec la conscience de son époque ; les paratextes, comme le titre, la dédicace, la préface, la postface, les notes du traducteur, etc.; enfin, le texte lui-même, avec ses erreurs, ses malentendus, ses phrases tronquées ou franchement supprimées. Autant d'indices qui pourraient davantage refléter la conscience d'une époque que les affirmations volontaires avancées par cette même conscience.

8 Pour mieux cerner le rapport entre idéologie et traduction, nous allons examiner les traductions de quatre textes marxistes. Les traducteurs des ces textes, qui appartiennent à des familles politiques différentes, ont réalisé ces traductions, animés de motivations variées.

\section{Ahmad Rif'at, le traducteur de L'État et la Révolution}

La première traduction en langue arabe d'un classique du marxisme fut l'ouvrage de Lénine, LÉtat et la Révolution, paru en janvier 1922 sous le titre Mudhakkirât Linîn 'an alhurûb al-urûbiyya: mâdîha wa hâdirihâ (Les mémoires de Lénine à propos des guerres européennes : leur passé et leur présent). Outre le titre, figure aussi sur la couverture du livre le nom du traducteur, Ahmad Rif'at et la mention suivante : «Par la plume de N. Lénine, président de la République russe ». Ahmad Rif'at est un nom qui n'a pas d'histoire dans la vie culturelle et politique égyptienne du $\mathrm{XX}^{\mathrm{e}}$ siècle ; il est absent du débat politique qui eut lieu dans la presse égyptienne à propos de la déclaration de la constitution du premier parti socialiste égyptien en 1921.

Les motifs pour lesquels Ahmad Rif'at a traduit L'État et la Révolution de Lénine ne relèvent pas d'un engagement théorique et politique pour le socialisme. On peut toutefois connaître sa conception de la Nahda et sa vision politique d'avant la guerre à travers une lettre où il répond aux revendications des coptes, et qu'il rédige après l'assassinat de Butrus Ghâlî, le premier ministre copte, et la tension qui s'en suivit entre coptes et musulmans. Rif'at affirme que l'Égypte est plus tolérante à l'égard des coptes que les pays occidentaux à l'égard des juifs; il conteste l'idée que les coptes seraient persécutés. Ce sont plutôt les musulmans, selon lui, qui représentent une majorité pauvre et opprimée, et qui ont le droit d'émettre des revendications. Il incite les 
Egyptiens à lutter contre la colonisation anglaise en maintenant le lien avec la Sublime Porte et à travailler ensemble pour la Nahda et le progrès (Rif'at, 1911, p. 31). Cette vision était à l'époque l'axe de la politique du Parti Watanî de Mustafa Kâmil et Muhammad Farîd.

11 Après la guerre, Rif'at a maintenu la même position, enrichie toutefois d'une ouverture sur les expériences des autres nations. Dans sa dernière traduction, al-Wataniyya al-'uthmâniyya (Le nationalisme ottoman, de B. G. Gaulis), parue également en 1922, il lance un appel aux jeunes d'Égypte dans lequel il précise sa conception de la Nahda: "L'Orient comme corps et existence spirituelle désigne un grand ensemble de pays et de peuples. Si ces pays se lient par une ligue, si ses fils se réunissent, il retrouvera la vie. » (Rif'at, 1922b, p. 3) Rif'at opte donc en 1922 pour «La grande ligue d'Orient ». C'était l'idée maîtresse d'al-Afghânî et d'Ishâq dans les années 1870 au sein de leur projet de la Nahda. Le panislamisme sera éclipsé en 1919 par le succès du Wafd qui soutenait un projet national égyptien sans préconiser l'unité avec les pays d'Orient. En Égypte cependant, le lien sentimental avec les pays d'Orient luttant contre le colonialisme restait populaire. Cette sensibilité devait se manifester lors des déclarations anticolonialistes de la révolution bolchevique et pendant le mouvement nationaliste turc.

Rif'at exprime cet enthousiasme dans sa traduction de l'État et la Révolution et du Nationalisme ottoman. Il félicite les bolcheviques pour leurs efforts de libération de l'Anatolie et leur soutien à la Turquie, et considère que le Congrès des peuples d'Orient à Bakou est un espoir pour l'unité et la renaissance des peuples musulmans. Dans Le Nationalisme ottoman, il présente l'exploit de Kemal Atatûrk et d'Enver Pacha comme un modèle à suivre par les Égyptiens. Entre les deux héros turcs, il penche du côté d'Enver Pacha, « héros musulman » qui «travaille pour le monde musulman en particulier, et l'Orient en général » (Rif'at, 1922b, p. 5). Il faut rappeler qu'Enver Pacha était à la tête d'une délégation représentant la Turquie, l'Égypte et la Tunisie au Congrès de Bakou (el-Makhadmeh, 1987, p. 92).

13 L'article intitulé "Socialisme, bolchevisme et religion», paru dans la revue fondamentaliste musulmane al-Manâr, témoigne de cet enthousiasme ${ }^{2}$. La manière dont Rif'at décrit Lénine montre ce que ce dernier représente pour un admirateur panislamiste; Rif'at présente Lénine au lecteur comme «le plus grand homme actuel sur le globe » (Rif'at, 1922a, p. 3). Lénine fut pour lui le modèle du gouvernant juste, modeste et dévoué dont l'Égypte avait besoin ; "Lénine, écrit-il, est un homme d'une extrême simplicité dans son alimentation, son habillement et sa demeure, il n'a d'autres préoccupations que de propager ses principes qui lient la libération mondiale de l'impérialisme à la réalisation de l'égalité entre tous les hommes » (Rif'at, 1922a, p. 5). La notion d'individu, héros sujet de l'histoire, est un concept principal dans le panislamisme, lequel vante l'exploit d'un seul homme (Mahomet). La solution des problèmes sociaux dépend de la personnalité du gouvernant. En outre, la présentation de Lénine en tant que héros, acteur de l'histoire, éclipse le rôle du parti, du marxisme, de la Nie Internationale et de la masse ouvrière.

Dans ce contexte, le choix de traduire L'État et la Révolution nous paraît circonstanciel. C'était peut-être à cette époque en Égypte le seul livre de Lénine traduit en français et accessible à Rif'at. Le changement du titre en Mémoires de Lénine sur les guerres européennes, leur passé et leur présent, permet à la fois d'éviter le mot thawra (révolution) et d'ajouter un titre accrocheur en raison de l'actualité de la guerre. Le choix du terme 
"Mémoire » est justifié par le projet du traducteur de présenter aux lecteurs égyptiens une série de livres sur « les grands esprits qui pensent clandestinement pour libérer leurs peuples " (Rif'at, 1922a, p.1) - série qui avait commencé par les Mémoires de Hindenburg. On peut saisir sa conception du socialisme en général et de la pensée marxiste en particulier dans la préface et la postface des Mémoires de Lénine. On peut déceler, dans la préface datant du 28 décembre 1921, de l'admiration pour la personnalité héroïque de Lénine. Près d'un mois après avoir envoyé le livre à l'imprimerie, il trouve utile d'écrire une postface intitulée "clarification nécessaire ", dans laquelle il explique certains concepts propres à la pensée socialiste, et commente la propagande antibolchevique dans la presse égyptienne et les derniers événements de la révolution russe.

D'après la postface, le communisme pour Rif'at signifie en même temps révolution et république. Il établit le lien entre les capitalistes et le régime monarchique, et ne conçoit pas le lien entre le communisme et la classe ouvrière. Rif'at voit dans le rétablissement de la monarchie après la révolution de 1848 et dans la chute de la Commune de Paris une confirmation de cette conclusion. La révolution bolchevique échappera-t-elle à ce destin impitoyable ? Rif'at ne laisse pas à l'avenir la tâche de répondre à cette question. La réponse, pour lui, réside dans la pratique politique du gouvernement bolchevique. Dans une sorte d'autocritique, Rif'at décrit d'abord la réception enthousiaste de la Révolution russe par les Orientaux. "Les bolcheviques sont apparus, au début, sous cet aspect noble qui les montrait au travail pour libérer les classes opprimées et les peuples soumis au joug du colonialisme [...]. Ils ont toléré la constitution de petites républiques en Ukraine, Finlande et dans le Caucase. Les âmes se sont rassurées. Les Orientaux en particulier ont pensé que la nature humaine de ces gens avait changé et que le rêve devenait réalité, leur bonne foi s'est étendue jusqu'à croire qu'ils allaient sauver le monde du cauchemar du colonialisme. Les Orientaux attendaient partout que le courant bolchevique atteigne leurs pays pour les sauver de l'emprise de ce cauchemar » (Rif'at, 1922a, p. 248).

16 Les Orientaux espéraient du bolchevisme une libération nationale plutôt qu'une justice sociale. L'enthousiasme de Rif'at pour Lénine dans la préface du livre est justifié par le soutien du gouvernement bolchevique au mouvement nationaliste turc; la déception de la postface est provoquée par les derniers événements : « Oui, le régime des Soviets nous a surpris ces derniers jours, par la suppression des républiques caucasiennes et l'occupation de ces pays par l'armée bolchevique, [...] alors que ces petites républiques étaient fondées sur le système communiste. » (Rif'at, 1922a, p. 249) Le rêve brisé n'est pas seulement celui des Orientaux, mais aussi celui du peuple russe, puisque les promesses du communisme - la suppression des différences entre les classes, l'abolition de tout pouvoir étatique et l'établissement d'une caisse générale qui reçoit la production et distribue d'après les besoins - sont loin d'être réalisées.

17 La critique du bolchevisme dérivée de la tendance panislamiste et anticolonialiste de Rif'at est loin d'être une critique fabriquée pour échapper à la censure, comme le suppose R. al-Safd qui affirme également que Rif'at était un membre du PCE, et que la traduction de ce livre est une contribution du Parti à la propagation de la pensée marxiste en Égypte (al-Saîd, 1976, p. 118). Cette hypothèse nous paraît peu justifiée car les idées politiques de Rif'at que nous venons d'exposer et le livre traduit n'ont jamais été discutés au sein du Parti et n'ont jamais constitué une source théorique pour la formation de ses cadres. Il suffira d'ailleurs de remarquer que le traducteur, trois mois 
seulement après la traduction de l'ouvrage de Lénine, opte pour le panislamisme en traduisant Le nationalisme ottoman.

Les motifs de la traduction sont donc l'enthousiasme, la curiosité et aussi la rentabilité marchande puisque Rif'at est un traducteur professionnel et que Lénine et ses idées sont un thème à la mode dans la presse égyptienne d'alors. Le titre choisi par le traducteur dévoile son approche du livre: il le considère comme un texte contre le colonialisme en soulignant la dénonciation par Lénine de la complicité des sociauxdémocrates avec leurs États bourgeois concernant l'engagement dans la grande guerre. Il résume le contenu du livre ainsi : d'abord, c'est un exposé des principes de la doctrine communiste fondée par Karl Marx et appuyée très fortement par son ami Engels; ensuite, le livre critique les idées des socialistes modérés et réfute leurs fausses thèses en les convainquant que le bonheur et le bien-être seront réalisés en nettoyant la terre des agents du colonialisme qui veulent soumettre les nations faibles aux anciens pouvoirs financiers autoritaires (Rif'at, 1922b, p. 4). Faute de connaissance de la théorie marxiste, la polémique principale de L'État et la Révolution, à savoir le rapport entre la classe ouvrière triomphante et l'État, échappe à la compréhension de Rif'at, ce qui marque négativement sa traduction. Toutefois elle reste la première tentative de traduction d'un texte marxiste et d'adaptation du vocabulaire et des analyses marxistes à la langue arabe. À ce titre, cette traduction mérite un examen détaillé3. Cet examen consiste, d'abord, à comparer cette traduction arabe de bonne heure de la terminologie marxiste à la traduction actuelle et à découvrir les erreurs éventuelles dans la traduction, afin de dégager leurs significations sociales et culturelles. Nous nous attacherons ici à l'examen du vocabulaire marxiste le plus important dans le texte: l'État, la révolution, la classe, la dialectique.

\section{État}

Rif'at traduit État par hukûma, un mot que l'on utilise dans la langue arabe actuelle pour désigner le gouvernement. L'État est traduit aujourd'hui par dawla qui signifie en arabe classique "puissance temporaire ». Rif'at utilise le mot dawla pour désigner les pays ou puissances colonialistes (duwal istïmâriyya). Utiliser hukûma pour traduire État était courant au XIX et au début du XX $\mathrm{XX}^{\mathrm{e}}$ siècles. À propos des phrases qui concernent le terme d'« État », les erreurs de Rif'at mènent parfois au contresens : Lénine écrit, citant Engels, «L'État n'est donc pas un pouvoir imposé du dehors à la société; il n'est pas davantage "la réalité de l'idée morale; l'image et la réalité de la raison" comme le prétend Hegel ». Rif'at traduit ainsi ce passage : « l'État n'est pas un pouvoir imposé en dehors du cercle de l'institution sociale; il n'a donc pas d'avantages, mais il est la réalisation d'une idée morale, il est image et résultat de la raison et de la vérité comme l'affirme Hegel. » (Lénine, 1972, p. 11 ; Rif'at, 1922a, p. 15) Rif'at, en confondant le sens du mot «davantage » avec "d'avantages » et en utilisant le verbe "être » là où se trouve le verbe "avoir", traduit le contraire de ce que Engels voulait dire. Autre exemple, dans la traduction française du texte de Lénine on lit : «Selon Marx, l'État est un organisme de domination de classe, un organisme d'oppression d'une classe par une autre; c'est la création d'un "ordre » qui légalise et affermit cette oppression en modérant le conflit des classes. » La traduction arabe donne : «De l'avis de Marx, l'État est un organe qui domine les classes, ou plus clairement, un organe d'esclavage d'une classe par rapport à l'autre. C'est lui qui a créé un ordre des choses et il l'a légalisé, et en vertu de cela, il a mis un terme à cet esclavage en étendant le conflit des classes. » 
(Lénine, 1972, p. 12; Rif'at, 1922a, p. 17) Selon la traduction, l'ordre créé par l'État ne légalise pas l'oppression comme le disait Marx, mais vise la fin de l'esclavage.

\section{Révolution}

20 Le terme révolution est traduit par thawra. Concernant le concept principal du livre de Lénine, le traducteur commet deux erreurs qui rendent vague la conception léniniste de la révolution: a) Lénine cite Marx qui postule que la violence doit jouer un rôle révolutionnaire, sans être nécessairement une source du mal. La traduction donne: «Comme la révolution, la violence joue un mauvais rôle.» b) Lénine montre que la tendance social-démocrate a trahi l'idée de révolution violente que l'on trouve chez Marx et Engels. La traduction dit : "L'abandon de l'idée de révolution violente est une trahison à l'égard des tendances socialistes, nationalistes, kautskistes. » (Lénine, 1972, p. 31 ; Rif'at, 1922a, p. 41) Ces erreurs rendent incohérentes, dans le texte arabe, les positions de Lénine à l'égard de la social-démocratie. Elles illustrent également à quel point le traducteur est ignorant de l'actualité du mouvement socialiste et de ses tendances. Cette ignorance se manifeste plus nettement encore dans la note qu'il consacre à Engels et qui le présente sous le nom de Jean-Jacques Engil : un écrivain socialiste allemand né en 1741, mort en 1802 (Rif'at, 1922a, p. 13) ${ }^{4}$, ou encore, lorsque voulant parler de Kerenski, il le confond avec Trotski ${ }^{5}$.

\section{Classe}

21 La traduction courante aujourd'hui est tabaqa. Rif'at utilise ce mot mais aussi : hay'a (organisation, corps), zumra (cohorte, faction, horde), fi'a (catégorie), tâ'ifa (communauté). Pour la bourgeoisie, la transcription en usage actuellement (burjwâziyya) ne figure pas dans la traduction de Rif'at. Il reste perplexe devant ce concept qu'il traduit d'abord par wajâha wataniyya (notoriété nationale) puis, dans la même page, par al-tabaqa al-mâliyya (la classe financière). En troisième lieu, il choisit tabaqat al-a'yân (la classe de propriétaires fonciers); ce mot est le plus utilisé dans le texte de Rif'at mais il ne le sera pas de manière systématique, car on trouve plus loin d'autres traductions comme al-aghniyâ' (les riches), al-wujahâ' (les notables), ashâb ru'ûs al-amwâl (les détenteurs de capitaux). Parfois, il opte pour l'usage de deux mots ensemble : al-aghniyấ' wa-l-a'yân (les riches et les notables). Cette indécision devant le terme «bourgeoisie» et la difficulté d'exprimer son sens en langue arabe se manifestent plus encore quand il traduit ce seul terme par quatre mots dans la même phrase; nous lisons dans le texte français cette expression: "sans la bourgeoisie et contre la bourgeoisie». Rif'at la traduit par «sans les propriétaires fonciers et les riches et malgré les notables et les capitalistes " (Lénine, 1972, p. 40; Rif'at, 1922a, p. 52). Rif'at donc n'a pas saisi le sens que Lénine donne au concept de «bourgeoisie » car il n'est pas un familier de la littérature socialiste. Cette difficulté de trouver un terme en arabe qui corresponde au concept de «bourgeoisie " peut être due à l'ordre social flou de l'Égypte dans lequel la classe dominante n'a pas un caractère précis. Nous pouvons toujours constater que les mots «notables » et " propriétaires fonciers » sont utilisés quand le texte d'origine évoque le rapport entre la bourgeoisie et le pouvoir d'État, et les mots « capitalistes » et «financiers » sont utilisés quand le texte évoque le conflit entre la bourgeoisie et la classe ouvrière. 
22 En ce qui concerne la petite bourgeoisie, la confusion est encore plus grande, ce concept désignant une classe non identifiable dans l'Égypte d'alors. Dans la littérature socialiste d'expression arabe, à cette époque, apparaît l'opposition qui existe dans la société entre riches et pauvres ou capitalistes et ouvriers, mais le concept d'une classe petite-bourgeoise était quasi absent. En outre, dans le texte de Lénine, le terme " petitbourgeois » désigne parfois une classe sociale réelle, mais, plus souvent une certaine tendance socialiste (la social-démocratie). Cela justifie la perplexité de Rif'at devant ce terme, qu'il traduit de deux manières différentes. Pour traduire le sens le plus courant quand Lénine dénigre la social-démocratie -, il utilise al-fi'a al-akthar wajâha (le groupe de grands notables), jahâbidhatal-a'yân (l'habileté des propriétaires fonciers), siyasiyu alfi'a al-mutanâhiya fi-l-wajâha (les politiciens du groupe des grands notables). Alors qu'il s'en tenait très éloigné avec le premier, Rif'at se rapproche, avec le deuxième sens, du concept léniniste de la "petite bourgeoisie ». On trouve pour la première fois cette traduction lors d'un passage où Lénine distingue la bourgeoisie de la petite bourgeoisie: "La bourgeoisie fractionne et dissémine la paysannerie et toutes les couches petites bourgeoises. » Ici Rif'at utilise al-tabaqât al-wûsta (les classes moyennes) et plus loin al-mullâk al-mutawassitûn (les propriétaires moyens) (Rif'at, 1922a, p. 16-17). En général l'attribut «petit-bourgeois" chez Rif'at est négatif et il implique l'appartenance à la bourgeoisie, il traduit donc «l'anarchie petite-bourgeoise » par « l'anarchie un peu aisée qui caractérise la vie de la classe moyenne » (Lénine, 1972, p. 39 ; Rif'at, 1922a, p. 50). Enfin, l'ambiguïté dans la traduction suscite des difficultés pour comprendre la position léniniste à l'égard de la social-démocratie, d'une part, et de la classe petite-bourgeoise, d'autre part.

\section{Dialectique}

Dans la traduction actuelle dialectique donne jadal. Rif'at, lui, traduit le terme par alqadâya al-mantiqiyya (les propositions logiques), ainsi, le "matérialisme dialectique » devient le " matérialisme logique », la « dialectique matérialiste » est rendue par madlûl hissî (sens concret), «le dialecticien Engels » devient «Engels le forgeron de mots ». Enfin « dialectique de l'histoire vivante » devient « la théorie logique qui est dérivée de la vie pratique » (Lénine, p. 79 ; Rif'at, 1922a, p. 100). Le mot «dialectique » lui est apparu énigmatique mais il l'a souvent traduit par «logique», ce qui est différent du sens de "dialectique ». C'est un autre type de fautes, dû au manque de connaissance philosophique. Il ne s'agit pas, ici, de recenser les fautes de traduction commises par le traducteur ou bien de dénoncer sa mauvaise foi, mais simplement de montrer combien il est difficile de comprendre les thèses de Lénine à partir de cette traduction comme de saisir le sens des débats entre Lénine et la social-démocratie. C'est pour cette raison que, dans la préface, Rif'at déclare qu'il a essayé de présenter « ces idées difficiles d'une manière simplifiée » (Rif'at, 1922a, p.2). Cette quête de simplicité l'a mené à ajouter volontairement ce qu'il trouvait nécessaire pour faciliter la compréhension. Par exemple, à propos de la situation des paysans, Lénine écrit: «[...] ces mesures démocratiques qui rendent parfaitement solidaires les intérêts des ouvriers et de la majorité des paysans"; Rif'at traduit: "[...] un moyen démocratique facile qui fonctionne d'une manière solidaire avec les intérêts des ouvriers et les intérêts de la majorité qui est la classe paysanne » (Lénine, 1972, p. 66 ; Rif'at, 1922a, p. 82). La réalité égyptienne se manifeste dans la traduction, non seulement lorsque l'on trouve cette affirmation de la paysannerie majoritaire, mais aussi à travers les conditions 
politiques; ainsi, quand Lénine dit: «La véritable besogne d'État se fait dans les coulisses, elle est exécutée par les départements et les états-majors ", Rif'at ajoute " les ambassades " dans la traduction (Lénine, 1972, p.69; Rif'at, 1922a, p. 87), faisant allusion à l'État égyptien dirigé par les ambassades étrangères, surtout celle de l'Angleterre.

La déformation du texte de Lénine est claire et nette quand il traite de religion, surtout quand Lénine critique le petit-bourgeois «libre penseur qui veut bien admettre que l'on ne soit d'aucune religion, mais abdique la tâche du parti, qui est de combattre l'opium religieux qui abêtit le peuple ». Rif'at rend cette phrase de Lénine en parlant de « la couche de la libre croyance qui accepte la neutralité de la religion et qui pourtant, en critiquant la campagne du parti contre les opinions religieuses, a anesthésié les nerfs du peuple » (Lénine, 1972, p. 113 ; Rif'at, 1922a, p. 145). La traduction a donc évité le mot «opium» et rendu responsable de l'abêtissement du peuple les idées de la couche de la libre croyance et non pas la religion.

Cette confusion dans la traduction a limité la portée du livre et l'a rendu énigmatique. C'est peut-être pour cette raison que Rif'at dans la publicité pour cet ouvrage parle d'un « livre merveilleux, étrange, étonnant, où l'intelligence se perd» (Rif'at, 1922b, p. 5). La presse hostile au bolchevisme en Égypte, en se rendant compte que le livre est traduit hors du champ de la lutte socialiste, ne critique plus le livre et n'y fait aucune allusion. L'attitude est d'ailleurs la même chez les socialistes. Le fait de traduire un ouvrage de Lénine montre quel avait été l'enthousiasme de Rif'at mais son commentaire de la postface, si critique à l'égard du bolchevisme, exprime une grande déception : cette déception est aussi celle de l'opinion publique égyptienne qui a déjà compris que le bolchevisme n'allait pas apporter la liberté tant attendue, mais, au contraire, poursuivre la politique colonialiste telle qu'elle fut menée sous le Tsar.

\section{Rashîd al-Barawi, le traducteur du Capital}

Rashîd al-Barâwî, professeur d'économie à l'université Fu'âd I, est le premier à traduire Le Capital en langue arabe, en 1947. Cette traduction paraît au moment où le communisme occupait la presse égyptienne d'une manière aussi passionnée qu'après la révolution bolchevique. À l'exception de quelques livres d'initiation au socialisme et d'articles favorables ou défavorables à l'Union soviétique, la pensée marxiste ne s'est présentée aux Égyptiens que par la traduction de L'État et la Révolution, de diffusion très restreinte, et par Travail salarié et capital, publié en 1930, en sept livraisons, dans un hebdomadaire. Il faut toutefois rappeler que les traductions faites par les groupes marxistes restent clandestines. Al-Barâwî justifie la traduction du Capital par des raisons académiques et par l'importance de cet ouvrage dans la recherche scientifique. Il présente le livre en disant : «Voici le livre intitulé Le Capital du célèbre savant Karl Marx. Nous le mettons entre les mains de l'élite des intellectuels arabophones qui visent une recherche scientifique approfondie et l'étude assidue de toutes les questions et doctrines économiques à partir de leurs références originales, pour mieux les comprendre. » (al-Barâwî, 1970, préf.) ${ }^{6}$

La contribution d'al-Barâwî à la propagation des textes marxistes ne se limite pas au Capital; il traduit un grand nombre de ceux-ci dans l'ordre chronologique suivant :

1. 1945 : L'Économie politique : questions et réponses de Léontieff;

2. 1946 : L'Impérialisme, stade suprême du capitalisme de Lénine ; 
3. 1947: Le Capital de Marx;

4. 1947 : Al-Tafsîral-ishtirâkîli-l-târîkh (L'interprétation socialiste de l'histoire), qui contient la traduction de : a) Ludwig Feuerbach et la fin de la philosophie classique allemande d'Engels ; b) la préface de l'édition anglaise de Socialisme utopique et socialisme scientifique d'Engels; c) la correspondance entre Marx et Engels sur le thème du matérialisme historique ; d) un résumé du livre La lutte des classes en France de Marx;

5. 1957 : Contribution à la critique de l'économie politique de Marx. relative de la valeur, où Marx compare la valeur de la toile à la valeur du vêtement, alBarâwî supprime la métaphore relative à " la nature moutonnière du chrétien dans sa ressemblance avec l'agneau de Dieu» (arabe, p. 17 ; anglais, p. 52 ; français, p. 581) ${ }^{8}$. Il supprime aussi cette phrase lucide de Marx : «Le monde religieux n'est qu'un reflet du monde réel» (ar., p. 42; an., p. 79; fr., p. 613). Al-Barâwi, pour éviter toute attaque franche contre la religion, modifie même parfois le texte; ainsi, quand Marx affirme qu'en général « le reflet religieux du monde réel ne pourra disparaître que lorsque les conditions du travail [...] présenteront à l'homme des rapports transparents et rationnels avec ses semblables et avec la nature", al-Barâwî traduit «le reflet religieux » par al-suwâr (les images) (ar., p. 42 ; an., p. 79 ; fr., p. 614). Tenant compte de ce que Marx disait dans le paragraphe précédent des anciennes religions, lesquelles renvoient, pour le lecteur arabe, à l'idée de paganisme, al-Barâwî utilise le mot 
«images" plutôt que l'expression "reflets religieux» pour orienter la pensée du lecteur vers les anciennes religions. La traduction a également subi des mutilations d'une autre nature. Al-Barâwî supprime les allusions philosophiques, comme le long passage dans lequel Marx explique le concept aristotélicien de la valeur (ar., p. 24 ; an., p. 58 ; fr.,p. 590). La note où Marx compare la marchandise au Moi fichtien subit aussi le même sort (ar., p.17; an., p. 52 ; fr., p. 582). Al-Barâwî, dans sa traduction, considère tout ce qui n'est pas économique comme hors sujet; c'est ainsi qu'il supprime l'analogie empruntée à la chimie, par laquelle Marx illustre la forme relative de la valeur (ar., p. 16 ; an., p. 50 ; fr., p. 579), ainsi que beaucoup de proverbes et dictons9.

Pour situer la traduction des textes marxistes par al-Barâwî, il faut repérer dans l'ensemble de son œuvre propre sa conception du développement de la société égyptienne et sa position vis-à-vis des courants politiques des années quarante et cinquante. Dans al-Tatawwur al-iqtisâdî li-Misr al-hadltha (L'évolution économique de l'Égypte moderne), publié en 1939, al-Barâwî consacre un chapitre à l'évolution de la classe ouvrière et souligne la nécessité d'accorder des droits, comme le droit de grève et l'interdiction du licenciement arbitraire, justifiant ces réformes par le fait qu'elles sont une garantie contre la radicalisation du mouvement ouvrier (al-Barâwî, 1944, p. 95-106) ${ }^{10}$. La traduction s'est donc effectuée dans un contexte non révolutionnaire, puisqu'elle ne vise ni à assister les ouvriers dans leur lutte pour la conscience révolutionnaire, ni à affronter la tradition religieuse, ni à prôner une société nouvelle. La traduction du Capital est dédiée à " ceux qui sacralisent la recherche scientifique... et la liberté bien guidée de l'opinion». Elle a donc un intérêt plus scientifique que politique (al-Barâwi, 1970, préf.).

Néanmoins al-Barâwi implique sa traduction du Capital dans sa vision du système adéquat à venir pour l'Égypte. De ce système, il dit qu'«il est, unanimement, pour ses partisans ou détracteurs, l'analyse scientifique la plus exacte du système capitaliste en son début, du rôle qu'il a joué dans le progrès, des contradictions qu'il contient et qui entraînent sa disparition au profit d'un système plus évolué » (al-Barâwî, 1970, préf.). Ce système plus évolué n'est ni le socialisme ni la dictature du prolétariat. Il explique sa conception du nouveau système en disant que «les marxistes, au-delà de leurs différends, voient dans le socialisme le système de l'avenir. Mais récemment, une théorie de l'écrivain James Burnham a nié cette probabilité et tend à considérer que les technocrates sont la classe dominante dans la société qui succède au capitalisme et qui se base sur la propriété par l'État des instruments de production "(al-Barâwi, 1970, préf.). La classe ouvrière, elle, ne fait que lutter pour améliorer ses conditions de travail au sein d'une société qui se transforme par la technologie.

En appliquant cette vision à la société égyptienne, al-Barâwî dessine un plan de la Nahda caractérisé par la lutte contre le colonialisme; le réveil des peuples arabes; l'économie mixte, nationalisée et privée; le rôle progressiste de la religion; l'amélioration des conditions de travail des ouvriers et des paysans. Son itinéraire intellectuel illustre cette conception de la Nahda. Nous avons déjà évoqué sa participation à la réforme des lois des syndicats en 1939, donnant des avantages aux ouvriers pour les éloigner du communisme. Ses écrits économiques d'avant 1952 réclament la nationalisation et font l'éloge de la politique du Parti du travail britannique. En outre, en 1952, quelques jours après le renversement de la monarchie par l'armée, il publie Haqîqat al-inqilâb al-akhîr fi Misr (La vérité sur le dernier coup d'État en Égypte), un livre plein d'enthousiasme pour le mouvement de l'armée : il le 
considère comme une "nécessité sociale » et trace les lignes de la politique du nouveau régime à l'échelle locale, arabe et internationale (al-Barâwî, 1952). Par la suite, le lien se renforce entre al-Barâwî et le nouveau régime. Il joue un rôle important dans de nombreuses entreprises nationales et, en même temps, il devient un des théoriciens du régime. En 1955, il écrit al-falsafa al-iqtisâdiyya li-l-thawra (La philosophie économique de la révolution). Cette philosophie inspirée du programme du Parti du travail britannique se résume en quatre points: $1^{\circ}$ ) la démocratie économique qui signifie l'actionnariat populaire ; $2^{\circ}$ ) la disparition des grosses fortunes par la modification du système fiscal ; $3^{\circ}$ ) le contrôle économique à travers les plans quinquennaux qui n'exigent pas que le système soit socialiste pour leur exécution ; $4^{\circ}$ ) l'élargissement des services et des fonctions économiques de l'État (al-Barâwî, 1955, p. 157-171).

Les idées exposées dans l'œuvre d'al-Barâwî n'ont pas trouvé un accueil enthousiaste parmi les intellectuels marxistes: en effet, ce dernier occulte les aspects révolutionnaires, s'adresse toujours à l'État et ne compte pas sur le rôle d'un mouvement populaire. Il se présente d'ailleurs comme un technocrate réformiste et évoque toujours le marxisme en l'insérant dans un cadre académique, le privant ce faisant de son rôle populaire. Il ne fait pas allusion à ces prétentions à transformer le monde ou à bouleverser la philosophie ou l'économie, ce qui, en retour, justifie l'indifférence des partisans du marxisme, comme de ses ennemis d'ailleurs, à l'égard de son œuvre. Le débat agité dans la presse sur le communisme a bien créé une curiosité chez les lecteurs éloignés des cercles marxistes, mais cette dernière a pu être assouvie par l'oeuvre disponible d'al-Barâwî.

\section{La traduction militante}

'Abd al-Fattâh al-Qâdî, après son retour d'Allemagne où il a étudié la médecine, fonde, en 1930, la revue Rûh al-'asr, pour propager la pensée socialiste. Il traduit la préface de la Contribution à la critique de l'économie politique et Travail salarié et capital. La revue sera interdite par ordre du Conseil des ministres dans la même année. Al-Qâdî forme un groupe constitué d'anciens militants du Parti communiste égyptien (PCE), interdit en 1926. Ce groupe restera sans activité mais aussi sans publication. En 1943, ses membres rejoignent l'organisation d'Henri Curiel, le Hamitu (al-Haraqa al-misriyya li-l-taharrur al-watanî ; en français : Mouvement égyptien de libération nationale, MELN), où al-Qâdî devient chef du bureau des intellectuels chargé de la traduction de textes fondamentaux du marxisme. En collaboration avec Fawzî Girgis qui, par son intermédiaire, deviendra lui aussi membre du MELN, al-Qâdî contribuera largement à la traduction de ces textes. Girgis résume ainsi les motifs de cette traduction : "Ce travail exprime notre sentiment à propos de la nécessité de l'éducation du «cadre égyptien ».» (al-Sa'îd, 1976, p. 362) Ce travail clandestin a produit treize ouvrages, publiés dans une collection passée à la postérité sous le nom de Majmû'atal-Kutub alkhadra (collection des livres verts) ${ }^{11}$, et vendus de manière semi-légale à la librairie alMî̀ân d'Henri Curiel, dont :

1. al-Bayân al-shuyû'î (Le manifeste communiste) ;

2. al-Ugûr wa-l-athmân wa-l-arbâh (Salaires, prix et profits), de Marx ;

3. al-'Amal al-ma'gûr wa ra's al-mâl (Travail salarié et capital), de Marx ;

4. al-Ishtirâkiyya al-'ilmiyya wa-l-lshtirâkiyya-l-khayâliyya (Socialisme scientifique et socialisme utopique), d'Engels ; 
5. Ta'âlîm Karl Marx (Les enseignements de Karl Marx), de Lénine ;

6. Ila al-rîf al-faqîr (lespaysans pauvres), de Lénine ;

7. al-Mâddiyya al-jadaliyya wa-l-mâddiyya al-târîkhiyya (Le matérialisme dialectique et le matérialisme historique), de Staline ;

8. 'An al-tandhîm (De l'organisation), de Staline ;

9. Usus al-lîniniyya (Les fondements du léninisme), de Staline ;

10. al-Mas'ala al-wataniyya wa-l-musta'marât (La question nationale et les colonies), de Staline ;

11. al-Iqtisâd muharrik al-târîkh (L'économie, moteur de l'histoire), de R. Garaudy. de former les cadres plutôt que d'éclairer le grand public. On a choisi principalement des livres d'initiation, simples, petits, pratiques dans les conditions de la clandestinité. Pour les militants égyptiens, ce sont des livres qui donnent accès à la vérité, à la théorie révolutionnaire et dont les communistes conçoivent le manque. Pour cette raison, le souci de la précision devient maniaque, comme le décrit Girgis : "La traduction était exacte, soigneusement révisée par des spécialistes en langue arabe et en langue anglaise, nous avons même eu recours à quelques membres de l'académie de la langue arabe. » (al-Sa'îd, 1976, p. 355) de la traduction de Salaires, prix et profits de Marx, le livre prend sa place dans ce programme : «La classe ouvrière égyptienne trouvera dans cet ouvrage de l'éducateur du prolétariat Karl Marx, ainsi que dans tous ses ouvrages, ce qui l'incite à réussir dans sa tâche historique : guider le peuple égyptien pour chasser le colonialisme et écarter ses agents, arracher sa liberté, établir la paix, constituer la république démocratique égyptienne, sur le chemin du socialisme puis du communisme» (Marx, 1954a). Ainsi, cette traduction s'adresse à un lecteur déjà engagé. Selon les témoignages de plusieurs militants, surtout en province, ces livres ont joué un rôle important dans leur engagement. La traduction sera interrompue en 1946, suite au grand procès du communisme qui entraînera la fermeture de la librairie al-Mîdân. Nous prendrons comme exemple de la collection verte, Le Manifeste du Parti communiste. La traduction commence par un exergue de Staline, «Le Manifeste du Parti Communiste est le Cantique des Cantiques du marxisme » (Marx, 1954b, p. 1), et par la préface d'Engels à l'édition allemande de 1883 et à l'édition anglaise de 1888. Dans la traduction, l'allusion que Le Manifeste fait à l'universalisation du mode de production capitaliste et à ses conséquences sur le nationalisme, est l'objet de plusieurs modifications.

39 - Dans la phrase : «En exploitant le marché mondial, la bourgeoisie a donné une forme cosmopolite à la production et à la consommation de tous les pays ", le traducteur traduit d'abord «le marché mondial» par les «marchés du monde » et au qualificatif « cosmopolite» il substitue "hétérogène» (Marx, 1954b, p. 19; 1965b, p. 156). Ces modifications présentent l'effort de la bourgeoisie pour universaliser le marché comme réactionnaire et contre nature.

40 - À la fin du même paragraphe, la phrase «les limitations et les particularismes nationaux deviennent de plus en plus impossibles » est traduite par « la pensée bornée et la fierté nationale deviennent jour après jour impossibles " (Marx, 1954b, p. 19; 1965b, p. 156). Ainsi, la portée de la remarque du Manifeste est réduite à un certain état d'esprit plutôt qu'à une réalité socio-économique.

Égypte/Monde arabe, 30-31 | 1997 
41 - À propos de la propriété, Le Manifeste déclare que « l'abolition des anciens rapports de propriété n'est pas un trait particulier au communisme » mais, dans la traduction, nous lisons: "l'abolition des rapports actuels de propriété n'est absolument pas un trait particulier au communisme » (Marx, 1954b, p. 32 ; 1965b, p. 175). Alors que Le Manifeste parle de la position du communisme vis-à-vis des anciens rapports de propriété qui sont déjà abolis par la bourgeoisie, la traduction parle de la position du communisme vis-à-vis de la propriété actuelle et nie le fait que son abolition soit un trait objectif du communisme.

42 - Dans le même contexte, Marx qualifie la propriété bourgeoise moderne d'expression ultime du mode de production fondé sur «l'exploitation des uns par les autres ». Dans la traduction, cette exploitation devient celle « de la majorité par la minorité » (Marx, 1954 b, p. 32 ; 1965b, p. 175). Cette modification, qui vise la réalité égyptienne, a pour conséquence de mettre en évidence la revendication de la justice sociale plutôt que l'abolition totale de l'exploitation. À l'analyse de cette traduction, une première remarque s'impose: les traducteurs marxistes étaient imprégnés des sentiments nationalistes, à l'origine des modifications qu'ils ont apportées au texte de Marx et d'Engels.

43 Nous en venons maintenant à la traduction des Principes fondamentaux de la philosophie (al-Mabâdî al-asâsiyya li-l-falsafa). Ce livre de Georges Politzer fut traduit, en 1957, par un communiste égyptien, dans l'élan national suscité par la guerre de Suez. Il eut une grande influence sur les militants et sur les étudiants. Il avait l'avantage d'avoir été publié légalement et de posséder un titre politiquement neutre. En outre, son auteur ne faisait pas partie des figures connues du marxisme et l'organisation de l'ouvrage en forme de leçons était très didactique, ce qui en favorisera l'écho hors du cercle des militants communistes. Dans la préface, le traducteur attaque «la philosophie colonialiste maligne » qui tourne le dos à la réalité et il soutient «la philosophie qui invite à changer la réalité » (al-Mahdâwî, 1957, p.3). La préface et les notes du traducteur font porter leur critique sur la philosophie positiviste logique, propagée en Égypte par Zakî Nagîb Mahmûd.

En examinant la traduction, nous avons repéré trois types de modifications. D'abord des modifications contextuelles, qui visent à tenir compte du contexte égyptien. On lit dans le texte d'origine: "Ils meurent en héros pour que vive la France ». En tenant compte de l'indignation collective en Égypte contre la France, au lendemain de la guerre de Suez, le traducteur al-Mahdawî traduit donc cette phrase : "Ils meurent en héros pour leurs principes » (al-Mahdâwi, 1957, p. 9 ; Politzer, 1954, p. VI). Ensuite, viennent les modifications qui visent à éviter la critique directe de la religion. L'intérêt de celles-ci, outre qu'elles ménagent l'opinion publique, c'est d'épargner au mouvement communiste une bataille idéologique jugée secondaire. Nous lisons, par exemple, dans le texte original: "Les chefs de l'Internationale socialiste se placent ouvertement sous l'aile de la religion. » Al-Mahdawî traduit: "...sous le drapeau du christianisme» (al-Mahdâwi, 1957, p. 26; Politzer, 1954, p. 14). Le même texte, commentant la théorie de l'évolution, dit : «Il n'est plus possible aujourd'hui de donner crédit au mythe répandu par la religion depuis des siècles : Dieu créant, une fois pour toutes, des espèces qui ne varient pas. » Le traducteur utilise le mot khurâfât (légendes) à la place du mot religion. Dans la phrase subordonnée, il utilise le participe passé évitant de mentionner Dieu. Ainsi sa traduction devient: "Il n'est plus possible aujourd'hui que les gens donnent crédit au mythe répandu par les légendes depuis des 
siècles : des espèces créées, une fois pour toutes, et qui ne varient pas. » (al-Mahdâwi, 1957, p. 55 ; Politzer, 1954, p. 52)

La traduction de ce dernier texte supporte bien la première remarque que nous faisions à propos de la traduction du Manifeste: le sentiment nationaliste égyptien est derrière la plupart des modifications apportées au texte original. Par ailleurs, il nous paraitt nécessaire de faire une seconde remarque : au contraire des traductions non militantes, les travaux des marxistes égyptiens cernent mieux les nouveaux concepts et atteignent une certaine stabilité de vocabulaire. Mais, à partir des années cinquante et surtout après l'amélioration des relations entre l'État égyptien et l'Union soviétique, les livres édités à Moscou en langue arabe remplacent les traductions militantes et deviennent la source la plus importante pour aborder la littérature marxiste.

À l'examen de ces textes, nous pouvons remarquer que les traductions, malgré l'appartenance des traducteurs à des horizons idéologiques différents, portent la marque de leur engagement nationaliste. En effet, ce que les intellectuels progressistes égyptiens cherchaient à travers ces traductions, c'était un soutien à leur lutte pour la libération nationale plutôt qu'une assise pour la révolution prolétarienne. Cette étude n'avait pas pour objectif de dénigrer les traductions examinées pour en proposer une meilleure, mais seulement de souligner que, s'il s'est agi d'adapter le marxisme aux revendications de la libération nationale, la déformation qui en a résulté a largement occulté toute possibilité de discussion critique des idées marxistes telles qu'elles sont exprimées dans les textes d'origine.

\section{BIBLIOGRAPHIE}

ABDEL-MALEKAnouar, 1970, La pensée politique arabe contemporaine, Paris, Seuil.

AL-AFGHÂNî, 1942, Réfutation des matérialistes, trad. GOICHON A.-M, Paris, Librairie Orientale.

AL-BARÂWÎ R, ÏLÎSM. H., 1944, al-Tatawwur al-iqtisâdi fi misr fî-l-'asr al-hadith, Le Caire, Dâr alnahda al-misriyya.

AL-BARÂWÎ R. :

- 1952, Haqîqat al-inqilâb al-akhîr fi misr, Le Caire, Dâr al-nahda al-misriyya.

- 1955, al-Falsafa al-iqtisâdiyya li-l-thawra, Le Caire, Dâr al-nahda al-misriyya.

ISHÂQA., 1978, al-kitâbât al-siyâsiyya wa-l-ijtimâ'iyya, Beyrouth, Dâr al-talî'a.

LÉNINE, 1972, L'État et la révolution, Paris, Éditions sociales.

AL-MAHDÂWÎ I., 1957, al-Mabâdi'al-asâsiyya li-l-falsafa, Le Caire, al-Dâr al-misriyya.

EL-MAKHADMEH S., 1987, Contribution à la critique de la pensée marxiste dans le monde arabe, Thèse d'État, Grenoble II.

MARX K. (traductions) : 
-1946, Capital, a Critical Analysis of Capitalist Production, trad. MOORE S. et AVELIN E., Moscow, Foreign Languages Publishing House.

- 1954a, al-Ugûrwa-l-athmân wa-l-arbâh (Salaires, prix et profits), Le Caire, al-Haraka aldîmuqrâtiyya lil-taharrur al-watanî (Haditu).

- 1954b, al-Bayân al-shuyûl (Le Manifeste communiste), Le Caire, al-Haraka al-dîmuqrâtiyya li-ltaharrur al-watanî (Haditu).

-1965a, Le Capital, (dans Euvres, vol. 1), trad. ROY J., Paris, Gallimard-La Pléiade.

- 1965b, Le manifeste communiste (dans Euvres, vol. 1), trad. RUBEL M. et EVRARD L, Paris, Gallimard-La Pléiade.

-1970, Ra's al-mâl, trad. AL-BARÂWÎ, Le Caire, Dâr al-nahda al-misriyya.

Al-Muqtataf, septembre 1879, art. « Ta'âlîm al-nihîlist».

POLITZER G., BESS G., CAVIENG M., 1954, Principes fondamentaux de la philosophie, Paris, Éditions sociales.

RIPAT A. :

-1911, al-Radd 'ala matâlib al-aqbât, Le Caire.

- 1922a, Mudhakkirât linîn 'an al-hurûb al-urûbiyya : mâdîha wa hâdiriha [trad, de L'État et la révolution de Lénine], Le Caire, al-maktaba al-tijâriyya.

- 1922b, al-Wataniyya al-'uthmâniyya [trad, du Nationalisme ottoman de GAULIS B. G.], Le Caire, Dâr al-tibâ'a al-fanniyya.

AL-SA'ÎD R., 1976, Târikh al-munadhdhamât al-yasâriyya fî misr, 1940-1950, Le Caire, Dâr al-thaqâfa al-jidîda.

\section{NOTES}

1. Actuellement le mot shuyûiiyya est en usage pour dire communisme et idrâb pour désigner la grève. Quant à ishtirâkiyya, qui avait suscité l'objection des premiers socialistes, il reste en usage et devient une qualification officielle du régime égyptien dans les années soixante.

2. Voir la traduction de cet article dans Abdel-Malek (1970, p. 232-237).

3. Pour faire cette comparaison, nous nous référons à la traduction française du texte de Lénine, parue en 1972 aux Éditions sociales.

4. Il corrigera cette erreur dans la postface en présentant une biographie plus exacte d'Engels (Rif'at, 1922a, p. 242).

5. «Après le renversement du Tsar, Lénine, qui n'était pas avide de pouvoir, a laissé les autres gouverner, mais l'égoïsme s'est emparé de Trotski qui aurait pu devenir dictateur, si Lénine n'avait pas sauvé la situation en l'écartant du pouvoir. » (Rif'at, 1922a, p. 5)

6. On se référera ici à la troisième édition de la traduction d'al-Barâwî (intitulée $R a$ 's al-mâl), parue au Caire en 1970 aux éditions Dâr al-nahda al-misriyya.

7. La traduction du Capital en langue arabe par al-Barâwî ne mentionne ni l'éditeur, ni la langue du texte d'origine. Mais al-Barâwî fait suivre la traduction arabe des concepts marxistes de leurs équivalents en anglais. Sachant par ailleurs qu'al-Barâwî est un traducteur familier de cette langue, il ne fait guère de doute que l'ouvrage dont il s'est servi était en anglais. Dans notre travail de comparaison, nous nous référons à l'édition de Moscou en langue anglaise, qui était la plus répandue (Moore et Avelin, 1946). Pour indiquer au lecteur français les passages non 
traduits ou modifiés par al-Barâwî, nous nous servons de la traduction française par Joseph Roy parue en 1965 dans La Pléiade.

8. Pour indiquer les passages qu'al-Barâwî a écourtés ou modifiés, nous mentionnons successivement les traductions arabe, anglaise et française de nos ouvrages de référence, respectivement abrégées désormais en ar., an., et fr.

9. Nous avons repéré 15 passages supprimés dans la trentaine de pages qui forment le premier chapitre de l'édition anglaise du Capital.

10. On se réfère ici à l'édition de 1944 de al-Tatawwur al-iqtisâdi fi Misr fîl-'asr al-hadîth, parue au Caire aux éditions Dâr al-nahda al-misriyya.

11. Éditée initialement par le Hamitu (MELN), la fameuse collection des livres verts fera l'objet d'une réédition par le Haditu (al-Haraqa al-dîmuqrâtiyya li-l-taharrur al-watanî; en français, Mouvement démocratique de libération nationale, MDLN), après la fusion en 1946 du Hamitu et de l'Iskra. C'est à cette réédition du Haditu que je me réfère dans cet article.

INDEX

Mots-clés : Marx, marxisme, traduction

\section{AUTEUR}

\section{ANWAR MOGHITH}

Université de Helwan 